\title{
It's Time to Take Advantage of Robotic Assisted Simple Prostatectomy in Large Benign Prostatic Hyperplasia
}

\author{
Myong Kim¹ ${ }^{1}$, Yu Seob Shin² ${ }^{2} ;$ KSSMA RASP group \\ ${ }^{1}$ Department of Urology, Asan Medical Center, University of Ulsan College of Medicine, Seoul, ${ }^{2}$ Department of Urology, Chonbuk National \\ University Medical School, Research Institute of Clinical Medicine of Chonbuk National University-Biomedical Research Institute, and \\ Clinical Trial Center of Medical Device of Chonbuk National University Hospital, Jeonju, Korea
}

This is an Open Access article distributed under the terms of the Creative Commons Attribution Non-Commercial License (http://creativecommons.org/licenses/by-nc/4.0) which permits unrestricted non-commercial use, distribution, and reproduction in any medium, provided the original work is properly cited.

The surgical management of large benign prostatic hyperplasia $(\mathrm{BPH})$ remains a challenge. Surgical methods have evolved over the last three decades from the traditional open simple prostatectomy, to transurethral techniques including transurethral resection of the prostate, holmium laser enucleation of the prostate (HoLEP), and other vaporization methods, as well as laparoscopic simple prostatectomy, or robotic assisted simple prostatectomy (RASP). The recent report by Umari et al [1] is indeed interesting, and it may be helpful to choose a proper surgical procedure for large $\mathrm{BPH}$. This article is one of scarce studies which compared treatment outcomes between RASP and HoLEP in patients with large-sized BPH. In this study, a total of 81 patients underwent RASP, and 45 underwent HoLEP during 7-years period [1]. Both groups demonstrated comparable post-operative improvements in urodynamic parameters and subjective symptom scores. Perioperative outcomes including operation time, risks for transfusion were similar in both groups.
HoLEP is a minimally invasive procedure for lower urinary tract symptoms suggestive of BPH [2]. Moreover, HoLEP has been shown to be associated with a higher enucleated tissue weight per enucleation time in large prostates, and theoretically to be a size-independent procedure [3]. However, HoLEP in extremely large prostate is challenging procedures, even after the surgeon has overcome the learning curve [4,5]. Technical challenges including bleeding risk, complex surgical plane, disturbance of surgical field, and difficulties in manipulating the resectoscope, are frequently encountered during the HoLEP of large prostates [4,5]. Moreover, HoLEP does carry a risk of postoperative complications, including urethral stricture, incontinence. The incidence of urethral stricture and late iatrogenic stress incontinence after HoLEP has been reported as $1.2 \%$ to $7.3 \%$ and $0 \%$ to $2.4 \%$, respectively [3]. In real-life practice, the true complication rates might be greater than that of previous reports, based on how and when the diagnosis is made. The majority of those complica-

Received: Nov 18, 2018 Accepted: Nov 19, 2018 Published online Dec 21, 2018

Correspondence to: Yu Seob Shin (iD https://orcid.org/0000-0002-1126-3821

Department of Urology, Chonbuk National University Medical School, 20 Geonji-ro, Deokjin-gu, Jeonju 54907, Korea.

Tel: +82-63-250-1560, Fax: +82-63-250-1564, E-mail: ball1210@hanmail.net 
tions after HoLEP are likely due to the use of a larger diameter scope for morcellation, or its retrograde access for adenoma dissection which is adjacent to the internal sphincter. The risks for such complications following HoLEP might be prostate size dependent.

In this respect, we believe that there might be a domain for RSAP to intervene in the surgical treatment for large BPH. Most importantly, in RSAP, fine anterograde dissection of the prostatic adenoma via high definition vision system and tiny wristed instrument of robot, which mimics the finger dissection of open simple prostatectomy, enables to lessen the risks for the complications including urethral stricture or incontinence. Considering those potential benefits by the RASP procedure, we believe that the adoption of this procedure in large BPH is reasonable. And our study group is now establishing a prospective multicenter RASP cohort to investigate our hypothesis in the future, and we are always welcome our collaborators.

\section{Disclosure}

The authors have no potential conflicts of interest to disclose.

\section{Author Contribution}

Research conception \& design: all authors. Drafting of the manuscript: all authors. Critical revision of the manuscript: all authors. Approval of final manuscript: all authors.

\section{REFERENCES}

1. Umari P, Fossati N, Gandaglia G, Pokorny M, De Groote R, Geurts N, et al. Robotic assisted simple prostatectomy versus holmium laser enucleation of the prostate for lower urinary tract symptoms in patients with large volume prostate: a comparative analysis from a high volume center. J Urol 2017;197: 1108-14.

2. Kuntz RM, Lehrich K, Ahyai S. Does perioperative outcome of transurethral holmium laser enucleation of the prostate depend on prostate size? J Endourol 2004;18:183-8.

3. Shah HN, Mahajan AP, Hegde SS, Bansal MB. Peri-operative complications of holmium laser enucleation of the prostate: experience in the first 280 patients, and a review of literature. BJU Int 2007;100:94-101.

4. Kim M, Piao S, Lee HE, Kim SH, Oh SJ. Efficacy and safety of holmium laser enucleation of the prostate for extremely large prostatic adenoma in patients with benign prostatic hyperplasia. Korean J Urol 2015;56:218-26.

5. Kim M, Lee HE, Oh SJ. Technical aspects of holmium laser enucleation of the prostate for benign prostatic hyperplasia. Korean J Urol 2013;54:570-9. 